\title{
Poly(methylene blue) modified electrode sensor for haemoglobin
}

\author{
Christopher M.A. Brett ${ }^{\mathrm{a},{ }^{*}}$, György Inzelt ${ }^{\mathrm{b}}$, Vilmos Kertesz ${ }^{\mathrm{b}}$

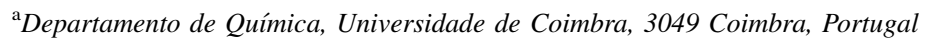 \\ ${ }^{\mathrm{b}}$ Department of Physical Chemistry, Eötvös Lorand University, Budapest 112, Hungary
}

Received 14 July 1998; received in revised form 6 November 1998; accepted 8 November 1998

\begin{abstract}
Poly(methylene blue) modified electrodes have been prepared by potential cycling from aqueous electrolyte solution containing methylene blue on glassy carbon substrates. They have been used successfully for the determination of haemoglobin via its electrooxidation at fixed potential using the batch injection analysis technique in which whole blood samples diluted with phosphate or acetate buffer are injected directly over the modified electrode immersed in inert electrolyte. Electrode response is fast, stable, and reproducible. For blood from healthy donors, a good linear correlation was found between the results from the electrochemical injection method and the conventional clinical spectrophotometric measurement after cyanidation. The blood from potentially ill donors showed deviations from the linear correlation suggesting that this may be a good method for detecting haematological problems. (C) 1999 Elsevier Science B.V. All rights reserved.
\end{abstract}

Keywords: Poly(methylene blue); Modified electrodes; Haemoglobin; Electrochemical sensor; Batch injection analysis

\section{Introduction}

Haemoglobin (HB) is an important protein in red blood cells, and in the reduced form, is the carrier of oxygen. It is also involved in a number of clinical diseases such as anemia, leukaemia and excessive loss of blood. Easy and accurate measurement of its concentration level in whole blood is thus extremely important. Current methods involve spectrophotometric determination after cyanidation or alkali hydroxylation; such methods are time-consuming and involve the use of highly purified and toxic reagents.

A direct electrochemical method based on the oxidation of reduced haemoglobin (iron (II) form) to methaemoglobin (iron (III) form) would be extre-

\footnotetext{
*Corresponding author. Fax: +351-39-835295; e-mail: brett@cygnus.ci.uc.pt
}

mely advantageous. As is the case for many large proteins, haemoglobin is irreversibly adsorbed on bare electrode surfaces leading to blocking and passivation. Suitable electrode modification is a way around this difficulty; the modifier layer acting as electron-transfer mediator and possibly catalysing the electron transfer process.

A number of studies have been carried out with this aim in mind. In one approach, haemoglobin has been studied at bare silver electrodes [1] and at silver electrodes modified by various surfactants. Although nearly all surfactants tested promoted the electron transfer, the best was found to be cetyl pyridinium chloride [2]. Further work has demonstrated that in such cases the electron transfer is not directly to the redox centre iron protoporphyrin IX but instead proceeds via complex formation of proteins with silver ions in the presence of chloride [3]. 
Haemoglobin direct electron transfer has also been observed in surfactant films of didodecyldimethylammonium bromide cast on glassy carbon electrodes at acidic and neutral $\mathrm{pH}$, and in the presence and absence of oxygen [4]. A mechanism for the electroreduction of methaemoglobin in these films has been proposed.

In another approach redox dyes have been employed as mediators. These have involved methylene blue [5-7], methylene green [8] and brilliant cresyl blue [9]. In all cases catalytic effects were observed. Regarding the methylene blue studies, in Ref. [5] spectroelectrochemistry was used to show the positive effects of methylene blue after adsorption on a platinum electrode at open circuit during $15 \mathrm{~min}$. A similar procedure was used in Ref. [6] at carbon fibre microcylinder electrodes, by immersion in methylene blue solution during $1 \mathrm{~h}$. Finally, in Ref. [7], methylene blue was mixed with Nafion solution and coated on the microcylinder electrode by dip-coating. This led to good results for the determination of haemoglobin in clinical blood samples. The Nafion film served to retain the methylene blue at the electrode surface.

It has been demonstrated that, as happens with a number of redox dyes, polymerisation of methylene blue occurs if a sufficiently positive potential is applied [10]. The formation of poly(methylene blue) by potential cycling has been studied using the electrochemical quartz crystal microbalance as has its redox behaviour as a function of $\mathrm{pH}$ and solution composition [11].

The objective of this work is to combine the enhanced electron transfer rate for haemoglobin oxidation at electrodes with adsorbed methylene blue, and which should be obtained at a poly(methylene blue) modified electrode, together with the batch injection analysis (BIA) technique [12]. This technique allows the measurement of microlitre volume samples by controlled injection from a micropipette held over the centre of an appropriate electrode immersed in electrolyte solution, with no necessity of electrolyte addition to the samples, unless dilution is required. It thus needs very little sample preparation and is a fast-response technique. It differs from flow injection analysis (FIA) [13]; in that there is no continuously flowing carrier stream. Besides the sample dispersion which arises between injection point and detector in FIA the continuous convection can also lead to problems of mechanical stability of the polymer films. Thus, although the experiments to be described were also carried out in an FIA mode, the BIA technique is to be preferred for use over a period of time. Therefore, the BIA technique is applied to the direct analysis of whole blood.

\section{Experimental}

The electrochemical cell contained a glassy carbon electrode (GCE), Pt gauze counter electrode and radiometer saturated calomel electrode (SCE) as reference. This arrangement was used for polymer film formation and for batch injection analysis, (BIA), in the special cell previously described [12].

In the BIA cell the micropipette tip (internal diameter $0.47 \mathrm{~mm}$ ) is held at a distance of $2-3 \mathrm{~mm}$ from the centre of the glassy carbon electrode (diameter 5 $\mathrm{mm}$ ). Injections were done from a Rainin programmable motorised electronic micropipette (EDP Plus 100), usually at the fastest dispensation speed of $74.5 \mu \mathrm{l} \mathrm{s}^{-1}$. The BIA cell was filled with inert electrolyte (usually $0.1 \mathrm{M}$ phosphate or acetate buffer).

All chemicals were of analytical grade and solutions were made with Millipore Milli-Q ultrapure water (resistivity $\geq 18 \mathrm{M} \Omega \mathrm{cm}$ ). Haemoglobin was obtained from Sigma and cytochrome- $c$ from Merck, and were used as received. Experiments were carried out using a BAS CV-50W potentiostat or an EG\&G PAR 273A Potentiostat/Galvanostat.

Human whole blood samples were obtained and immediately treated with EDTA anticoagulant (leading to a dilution of $10 \%$ ), according to standard procedure, and divided into two parts. One part was used for electrochemical experiments. The other was used for haemoglobin determination by the standardised protocol employing cyanidation in bicarbonate solution which converts all haemoglobin to cyanmethaemoglobin, followed by measurement of absorption at $540 \mathrm{~nm}$ [14]. Samples were stored at $4^{\circ} \mathrm{C}$ and analysed within $24 \mathrm{~h}$.

\section{Results and discussion}

Poly(methylene blue) (PMB) films were grown on the glassy carbon electrode. Fig. 1 shows typical 


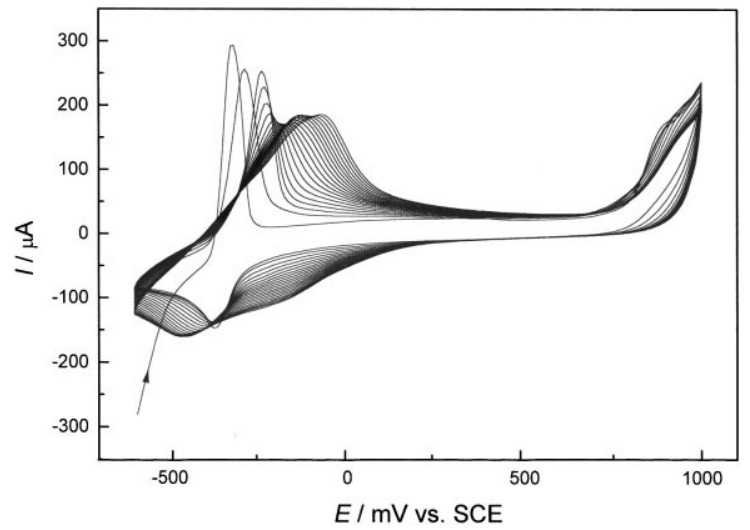

Fig. 1. Growth of poly(methylene blue) film on glassy carbon electrode substrate by potential cycling at a scan rate of $100 \mathrm{mV} \mathrm{s}^{-1}$. Solution contains $2.0 \mathrm{mM}$ methylene blue, $0.1 \mathrm{M}$ phosphate buffer ( $\mathrm{pH}$ 8.2) and $0.1 \mathrm{M} \mathrm{Na}_{2} \mathrm{SO}_{4}$.

cyclic voltammograms obtained during film growth. The voltammetric profile is similar to that obtained on gold [11]. The initial signal due to monomer at $\sim 0.3 \mathrm{~V}$ vs SCE decreases and peaks corresponding to the polymer increase with successive cycles. The optimised medium for polymer film growth was employed [11].

Electrodes were left to age in electrolyte for several hours, or overnight, before being used for haemoglobin determination. This allowed unpolymerised monomer retained within the film to escape and led to a more stable response. Before making any measurements, and periodically during series at measurements, the integrity of the PMB film was checked by cyclic voltammetry, the response being as in Fig. 2 .

In order to measure the oxidation of reduced haemoglobin, the electrode was held at $+550 \mathrm{mV}$ vs. SCE. The chronoamperometric response for consecutive injections of $50 \mu 1$ samples of whole blood after dilution in phosphate buffer is shown in Fig. 3. Dilution is advisable since concentrations of $\mathrm{Hb}$ in blood are of the order $2 \mathrm{mM}\left(130 \mathrm{~g} \mathrm{l}^{-1}\right)$. The form of the transient is as expected for BIA. The sudden drop in current and negative peak towards the end of the transients was due to removal of the micropipette from the cell. Between each injection of blood, a blank phosphate buffer electrolyte sample was injected. This was done to make absolutely sure that there are no memory effects. Although the wall-jet principle by which the BIA technique functions pre-

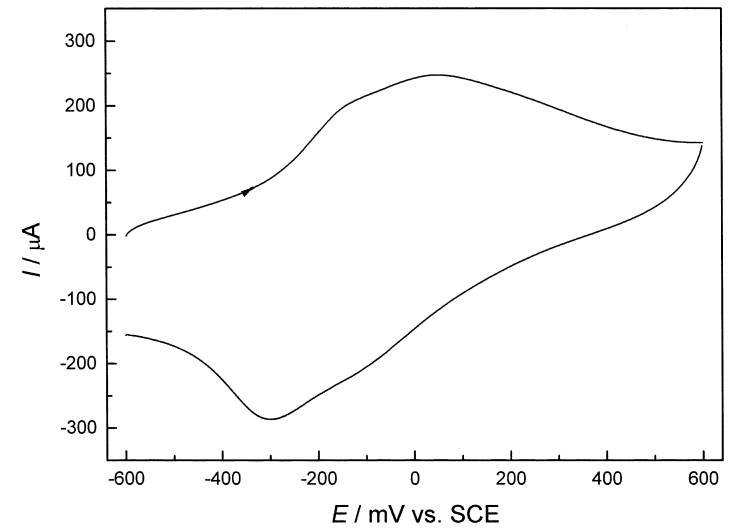

Fig. 2. Cyclic voltammogram of PMB electrode after aging $24 \mathrm{~h}$ in $0.1 \mathrm{M}$ phosphate buffer electrolyte, scan rate $=100 \mathrm{mV} \mathrm{s}^{-1}$.

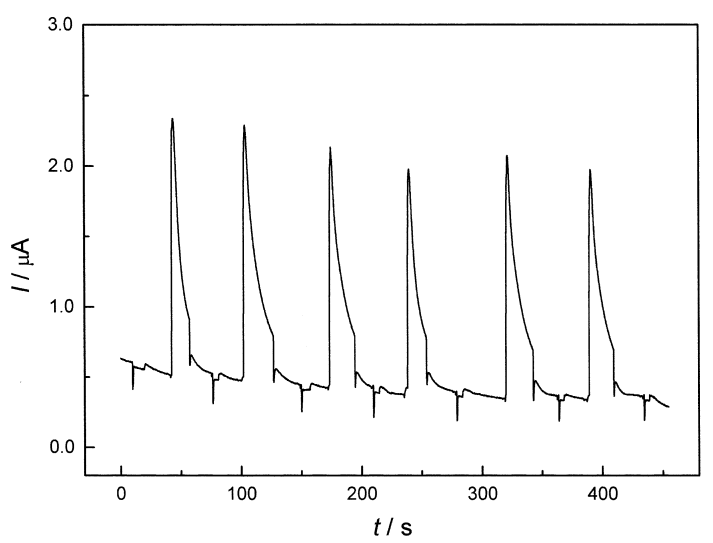

Fig. 3. Chronoamperometric response for consecutive injections in BIA cell of $50 \mu 1$ samples of whole blood (diluted 1:5 in phosphate buffer) on PMB electrode; $E=+550 \mathrm{mV}$ versus SCE.

cludes memory effects at modified electrode surfaces such as PMB, there could be some residual retention of adsorbed species which may contribute to the next injection of blood sample. Before injection of the next sample and after reintroduction of the micropipette, some $20 \mathrm{~s}$ were left to let the background current drop to a "stable" value.

Tests with commercial haemoglobin gave no distinguishable signal at such potentials, which can be attributed to the fact that it is almost $100 \%$ methaemoglobin. To test possible other proteins which may be oxidised at PMB, experiments were also carried out with cytochrome- $c$ chronoamperometrically or by cyclic voltammetry. Again, no signal was measured. 


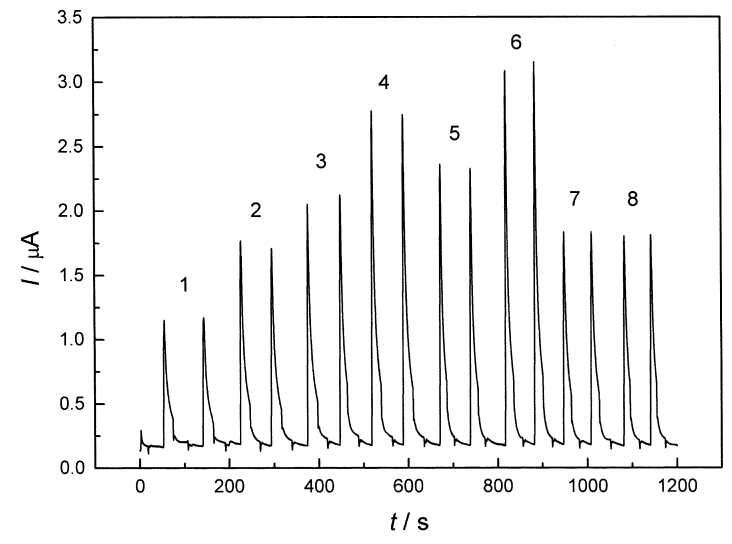

Fig. 4. Chronoamperometric transients for the analysis of blood samples 1-8. Experimental conditions as in Fig. 3.

In Fig. 4 are shown results from blood samples provided by eight donors. The responses are plotted as peak currents versus the cyanidation method results. Samples 1-4 refer to healthy patients and a good linear correlation between the electrochemical and cyanidation analysis methods can be observed. With respect to potentially ill patients, although deviation for sample types 7 and 8 from the correlation line are small, those from patients 5 and 6 differ significantly. This different ratio between electrochemical and cyanidation responses may therefore be useful in indicating whether the patient has a haematological problem in the blood.

The determinations referred to above were carried out in phosphate buffer at $\mathrm{pH}$ 8.2. This is above the isoelectric point for HB of 6.8 at which HB loses its protonation. It was thus felt interesting to carry out the same experiments at a lower $\mathrm{pH}$. A value of 5.0 was chosen; the blood being diluted in acetate buffer. Results obtained are shown in Fig. 5. As can be seen, there is also a good linear correlation for sample types 1-4. Confirmation that the results in the two media can be used as alternative procedures is given in Fig. 6, where it is shown that results from the two $\mathrm{pH}$ values all fall on a straight line.

For three replicate measurements a standard deviation of $5 \%$ can be estimated for the electrochemical oxidation of haemoglobin at PMB electrodes, which is perfectly acceptable for this type of determination. Regarding the detection limit, in the cyanidation method this is measured by successive dilution of haemoglobin standards until the absorption at

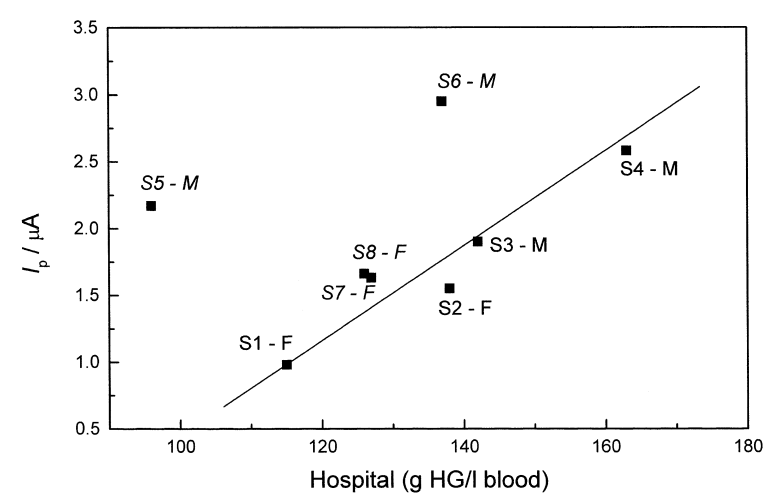

Fig. 5. Comparison between electrochemical (pH 8.2 phosphate buffer) and cyanidation methods for analysis of blood in patients 1$8(\mathrm{M}=$ male, $\mathrm{F}=$ female; patients $1-4$ healthy, patients 5-8 potentially ill).

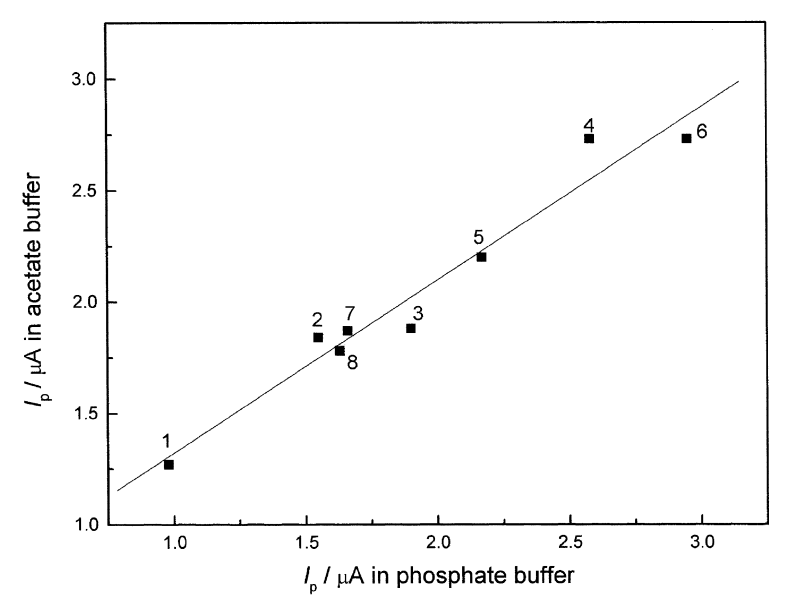

Fig. 6. Comparison between the BIA response after dilution of whole blood samples in phosphate buffer $(\mathrm{pH} \mathrm{8.2)}$ and in acetate buffer (pH 5.0).

$540 \mathrm{~nm}$ cannot be distinguished. The theoretical electrochemical detection limit can be estimated from the results in this work as equivalent to a signal of $0.05 \mu \mathrm{A}$ (three times the noise level) which corresponds to $0.25 \mathrm{~g} \mathrm{l}^{-1}$ from the correlation in Fig. 4, or a dilution factor of $\sim 100$ of the original blood sample. This is very far below any levels which are required to be measured in practice.

It has been pointed out, [4], that protons can influence the oxygen-carrying ability of ferrous haemoglobin according to

$$
\mathrm{HbFe}(\mathrm{II}) \mathrm{O}_{2}+\mathrm{H}^{+} \rightleftharpoons \mathrm{HbFe}(\mathrm{II}) \mathrm{H}^{+}+\mathrm{O}_{2}
$$


It is of interest that the measurement of $\mathrm{Hb}$ at $\mathrm{PMB}$ electrodes is not sensitive to this equilibrium.

Further studies are underway to clarify the reasons for the deviation from linearity with the spectrophotometric response for the determination of haemoglobin in blood from ill patients at PMB-modified electrodes. If such deviations are linked to structural alterations occurring in haemoglobin variants, then perhaps it could be used as a viable diagnostic alternative to mass spectrometry [15].

Additionally, it should be remembered that the electrochemical signal is proportional to the concentration of reduced forms of haemoglobin, whereas the spectrophotometric signal is proportional to the total concentration of all forms of haemoglobin. Thus, deviations could also be indicative of a larger proportion of reduced forms, and may be a good earlywarning probe for haematological problems in the blood.

\section{Acknowledgements}

We thank Dr. M. Horányi and A. Tücsök (Institute of Haematology, Budapest), Dr. G. Nagy (Health centre 11th District, Budapest) for supplying tested blood samples and for useful discussion, and the collaboration of Dr. F. Plácido and Dr. M. Vivan (Haematology Clinic, University of Coimbra Hospital).

The support of Instituto de Cooperação Científica e Tecnológica Internacional (ICCTI), Portugal (project 423/OFMB/SCIAE) and the National Technological
Development Committee (OFMB), Hungary (project TéT P-1/96) in the framework of the Luso-Hungarian Cultural Agreement and National Scientific Research Fund, Hungary (OTKA-TO14928) is gratefully acknowledged.

\section{References}

[1] G. Li, X. Liao, H. Fang, H. Chen, J. Electroanal. Chem. 369 (1994) 267.

[2] B. Ye, X. Zhou, Electroanalysis 8 (1996) 1165.

[3] M. Katterle, U. Wollenberger, F.W. Scheller, Electroanalysis 9 (1997) 1393.

[4] M. Cireanu, S. Goldstein, M.A. Mateescu, J. Electrochem. Soc. 145 (1998) 533.

[5] S. Song, S. Dong, Bioelectrochem. Bioenerg. 19 (1988) 337.

[6] H. Ju, J. Zhou, C. Cai, H. Chen, Electroanalysis 7 (1995) 1165.

[7] H.Y. Chen, H.-X. Ju, I.G. Xun, Anal. Chem. 66 (1994) 4538.

[8] Y. Zhu, S. Dong, Electrochim. Acta 35 (1990) 1139.

[9] S. Dong, Y. Zhu, S. Song, Bioelectrochem. Bioenerg. 21 (1989) 233

[10] A.A. Karyakin, A.K. Strakhova, E.E. Karyakina, S.D. Varfolomeyev, A.K. Yatsimirsky, Bioelectrochem. Bioenerg. 32 (1993) 35.

[11] V. Kertész, J. Bácskai, G. Inzelt, Electrochim. Acta 41 (1996) 2877.

[12] C.M.A. Brett, A.M. Oliveira Brett, L.C. Mitoseriu, Electroanalysis 7 (1995) 225.

[13] J. Ruzicka, E.H. Hansen, Flow Injection Analysis, 2nd ed., Wiley, New York, 1988.

[14] E. Beutler, Red Cell Metabolism. A Manual of Biochemical Methods, Grune and Stratton, New York, 1971.

[15] C.H.L. Shackleton, H.E. Witkowska, Anal. Chem. 68 (1996) 29A. 